\section{Adaptación del instrumento WAST para la detección de violencia doméstica en Centros de Salud}

\author{
LORENA BINFA ${ }^{1, \mathrm{a}}$, VALENTINA CANCINO ${ }^{1, \mathrm{~b}}$, ISAAC UGARTE ${ }^{1, \mathrm{~b}}$, \\ MARIBEL MELLA ${ }^{1, b}$, GABRIEL CAVADA ${ }^{2, \mathrm{c}}$
}

\section{Cultural adaptation and translation of the Woman Abuse Screening Tool}

\begin{abstract}
Background: The Woman Abuse Screening Tool (WAST) is a screening tool for domestic violence used in Unites States. Aim: To carry out the cultural adaptation of the WAST instrument for early detection of domestic violence in primary health care. Material and Methods: Qualitative techniques for cultural adaptation were used in stages 1 and 2 and quantitative techniques were used in stage 3. The validity of content was assessed using judge tests carried out with experts in the field. Linguistic adaptation was carried out using a focal group technique to ensure semantic and language comprehension. Finally, the culturally adapted instrument was applied to 16 women who were victims of violence and to 28 women without a history of violence. Results: For cultural adaptation, the judges' test added the item of economic violence to the instrument. According to the focus groups, some words were changed to facilitate understanding by the subjects. According to the scores obtained in women with and without a history of violence, a cut-off point of 15 points was defined to determine a history of domestic violence with a sensitivity of $100 \%$ and a specificity of $96 \%$. The Cronbach Alpha of the questionnaire was 91\%. Conclusions: WAST is an effective and easily applied instrument for the early detection of domestic violence.
\end{abstract}

(Rev Med Chile 2018; 146: 331-340)

Key words: Battered Women; Domestic Violence; Primary Health Care; Spouse Abuse; Survey and Questionnaires.

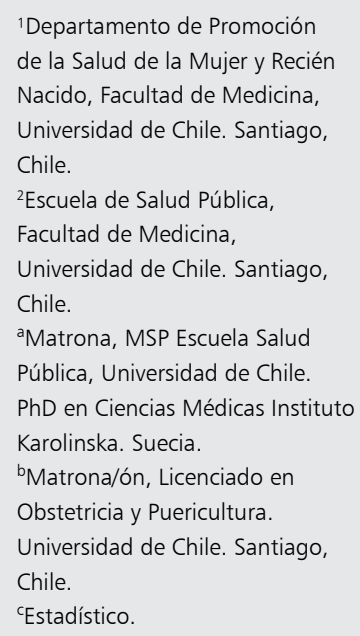

Recibido el 30 de junio de 2017 , aceptado el 28 de marzo de 2018.

Correspondencia a: Lorena Binfa Esbir Independencia 1027. Tel.: 229786619-229786618 Ibinfa@uchile.cl

\section{L} a violencia doméstica (VD) es un grave problema que aqueja a muchas mujeres y una transgresión a sus derechos humanos fundamentales ${ }^{1}$. A nivel global, según estimaciones de la Organización Mundial de la Salud (OMS), entre 10 y $69 \%$ de las mujeres han sufrido agresiones físicas por parte de una pareja y entre 10,4 y $30,3 \%$ violencia de tipo sexual, de acuerdo a la Organización de Naciones Unidas, casi la mitad de las mujeres víctimas de homicidio fueron a manos de sus parejas o un familiar ${ }^{2-4}$. En Chile se estima que una de cada tres mujeres es víctima de algún tipo de violencia ${ }^{5}$, cifras que se relacionan con lo reportado a nivel global, esta situación es preocupante, en nuestro país una mujer muere semanalmente víctima de este tipo de violencia ${ }^{4,5}$.

Tanto a nivel internacional como local se ha reconocido la gravedad de la $\mathrm{VD}$, la cual se define como "toda forma de maltrato o abuso físico, psicológico-emocional, sexual o económico que tiene lugar en la pareja", para connotar la importancia de la violencia contra las mujeres destaca dentro de las metas del objetivo 5 de los "Objetivos de Desarrollo Sostenible”, el cual tiene relación con lograr la igualdad entre los géneros, la relevancia de acabar con toda forma de discriminación y violencia ${ }^{6}$. 
Chile ha suscrito acuerdos internacionales y de orden jurídico con el objetivo de otorgar protección a la mujer. Un primer paso fue la creación de la Ley No 19.325 de violencia intrafamiliar, aprobada en 1995, posteriormente en 1999 se introdujo una modificación en el código penal relativa al abuso sexual, Ley $\mathrm{N}^{\circ} 19.609$ y en 2005, la Ley No 20.066 agrega normas sobre los deberes que corresponden al Estado en esta materia y la obligatoriedad de medidas de protección, modificándose en la Ley 20.480 de 2010 , en que se tipifica el femicidio y se aumenta la pena aplicable a este delito ${ }^{5}$, además, a partir de 2016 se crea el Ministerio de la Mujer y Equidad de Género (SERNAMEG), que tiene entre otras funciones "ejecutar modelos programáticos para prevenir, erradicar y sancionar la violencia contra las mujeres e intrafamiliar"7.

Sin embargo, todos estos esfuerzos se ven mermados por la restringida capacidad de una detección precoz de estos eventos. El contexto clínico, según la literatura, es idóneo para la detección de $\mathrm{VD}^{8,9}$, en atención primaria de salud (APS) es donde se abordan aspectos de promoción, prevención y detección temprana, constituyéndolo como un espacio propicio para satisfacer esta necesidad. A pesar que existen factores que dificultan la detección de violencia, los mismos estudios señalan que muchas mujeres están esperando que se les consulte al respecto, buscando ayuda y seguridad para detener la agresión ${ }^{10}$. El Estado ha participado de la elaboración de documentos que abordan la temática en APS, pautas indicadoras de VD y otra de riesgo de sufrir lesiones o femicidio ${ }^{11}$, sin embargo, estos no son instrumentos de tamizaje, quedando en manos de cada profesional cómo efectuarlos. Estudios señalan que la ausencia de instrumentos de tamizaje causa una subdetección de $70 \%$ de los casos, por lo que disponer de estos instrumentos constituiría una herramienta de gran utilidad ${ }^{8,9}$. Dentro del equipo de salud, un profesional que puede desarrollar esta detección es la matrona/ón, por ser quien otorga atención integral vinculándose con las mujeres, adicionalmente cubre gran parte de las actividades del Programa de Salud de la Mujer ${ }^{12}$, por lo que la implementación de un instrumento de cribado sencillo y rápido, disponible para todos los profesionales de APS, aumentaría esta posibilidad.

La finalidad de este estudio fue realizar la adaptación cultural y validación en población chilena del instrumento WAST (Woman Abuse Screening
Tool), originario de Estados Unidos de Norteamérica y validado al español ${ }^{13}$, el cual fue elegido por ser uno de los mejor evaluados por la guía inglesa para la detección de $\mathrm{VD}$, recomendando su uso en contextos no específicos de maltrato, como APS. Es corto, de fácil comprensión y ha demostrado tener buena consistencia interna en otros estudios que lo han utilizado, validado para población de habla hispana y de autoaplicación, modalidad que prefieren las usuarias ${ }^{13,14}$.

\section{Material y Método}

Estudio que constó de una primera fase cualitativa para realizar la adaptación cultural y lingüística del instrumento y una segunda fase cuantitativa, observacional y descriptiva para validar y determinar el punto de corte y rendimiento (sensibilidad y especificidad) del instrumento.

Para la validez de contenido ${ }^{15}$ se usó la técnica juicio de expertos ${ }^{16}$, con profesionales del entonces Servicio Nacional de la Mujer (SERNAM), Servicio Nacional de Menores (SENAME) y matronas docentes de la Universidad de Chile, el cual determinó que la palabra "abuso" del instrumento original es un término abstracto para referirse a las formas de violencia, se propuso modificar las siguientes preguntas: a) Abuso físico: Preguntar si las discusiones terminan en golpes, patadas, empujones; b) Abuso sexual: Preguntar si se han visto obligadas a tener relaciones sexuales; c) Abuso psicológico: Preguntar por el trato de sus parejas, si les gritan o hablan en forma grosera. Además, se decidió incorporar una nueva pregunta que evalúa la violencia económica, ya que es un aspecto de VD según la realidad nacional, para esto, se reemplazó la pregunta 6 , ya que esta preguntaba por el abuso físico, y se consideró que este aspecto ya se medía de mejor manera en la pregunta 4 . La adaptación lingüística se logró al conformar un grupo focal con 9 mujeres del personal auxiliar femenino de la Universidad de Chile. El rubro laboral en el que se desempeñan las participantes, su nivel socioeconómico, escolaridad y edad, entre otras, tienen características similares a la población donde posteriormente se aplicaría el instrumento.

En cuanto a la determinación de la muestra para el cálculo del punto de corte se estimó como universo a mujeres inscritas en el programa de la mujer en los centros de salud (CESFAM) de la 
zona norte de la Región Metropolitana, año 2015. El cálculo del tamaño muestral para determinar la capacidad diagnóstica del instrumento consideró un likelihood ratio positivo (LR+) igual a 14 (sensibilidad de $89 \%$ y especificidad de $94 \%$ ) $^{17}$, así para una significación de $1 \%$ y potencia de $99 \%$, se estimó un tamaño de muestra de 14 mujeres con certeza de violencia y 14 mujeres con certeza de no violencia, además, se consideró una prevalencia de violencia en la Región Metropolitana de 50,3\%

Las personas que conformaron la muestra sin violencia fueron mujeres inscritas en los CESFAM Dr. Patricio Hevia y Cristo Vive en el año 2015, comuna de Recoleta. Los criterios de inclusión fueron ser mayor de edad, asistir a consulta con la matrona y encontrarse en una relación de pareja de más de 6 meses, definido por los autores como punto medio, dado que varios instrumentos validados para este fin no consideran el tiempo de la relación como criterio de inclusión, y otros consideran un tiempo de un año $\mathrm{o}^{14,18}$. Los criterios de exclusión fueron mujeres diagnosticadas con trastornos de salud mental, relación homosexual, analfabetas y mujeres que acudían acompañadas por su pareja. Las mujeres con certeza de VD fueron contactadas en el Centro de la Mujer de la comuna de Conchalí, dependiente del entonces SERNAM, centro donde acudían mujeres víctimas de violencia para recibir asesoría legal y social, entre otras.

El instrumento utilizado fue el Woman Abuse Screening Tool (WAST) que consta de 8 preguntas tipo Likert que interroga sobre a) el grado de estrés existente en la relación; b) dificultad para resolver discusiones y c) existencia de episodios violentos a nivel emocional, psicológico, físico o sexual y cuyas posibles respuestas se disponen en tres niveles que van de menor a mayor gravedad: la respuesta menos deseable es evaluada con 3 puntos, la intermedia con 2 puntos y la más deseable con 1 punto; así, el máximo valor del puntaje es de 24 puntos y el mínimo de 8. Este instrumento fue traducido al español y validado por Forgarty y Brown en pacientes hispanohablantes de Estados Unidos de Norteamérica en el año 2002, obteniendo una sensibilidad de $89 \%$ y especificidad de $94 \%$, con un alfa de Cronbach de $0,91^{13}$.

En la fase de aplicación del instrumento WAST para la muestra de mujeres sin violencia, se invitó a participar a quienes acudían a control con matrona, que estaban en la sala de espera del
CESFAM, previo verificar cumplir con los criterios de inclusión y otorgamiento del consentimiento informado. Para la muestra de mujeres con violencia, los investigadores se contactaron con el Centro de la Mujer y realizaron la invitación a participar del estudio, luego de presentar los objetivos y otorgar su consentimiento informado, asegurando la voluntariedad y confidencialidad.

Para el análisis de datos cuantitativos se construyó una base de datos Excel para, posteriormente, ser analizados en el paquete estadístico Stata versión 13.0. Los puntajes finales de la aplicación del instrumento WAST se evaluaron a través del área bajo la curva de ROC, proponiendo el punto de corte al que se le determina mayor LR + , con el fin de que el instrumento tenga alta capacidad de detección de violencia ${ }^{19}$.

Este estudio fue aprobado por el Comité de Ética para la Investigación en Seres Humanos de la Facultad de Medicina de la Universidad de Chile y adicionalmente por el Comité de Ética del CESFAM Cristo Vive de la comuna de Recoleta.

\section{Resultados}

Respecto el resultado obtenido tras someter el instrumento a la prueba de expertos, se hizo el cambio de la palabra "abuso" en todos los ítems del instrumento reemplazándola por un concepto que facilitara su comprensión, se eliminó un ítem que se consideró reiterativo, reemplazándolo por incorporó una pregunta relativa a violencia económica, no considerado en el instrumento (Tabla 1).

Respecto los resultados posteriores a la realización del grupo focal, las participantes no refirieron problemas para comprender el lenguaje de las preguntas, aun así, realizaron 3 sugerencias que no alteran el significado global de las preguntas, por el contrario, facilitan la comprensión (Tabla 1).

Entre los principales resultados, destaca que la caracterización sociodemográfica de las participantes mostró que las mujeres pertenecientes al CESFAM y al SERNAM se encontraba, en su mayoría, en una relación de pareja. Respecto a la ocupación, en el grupo de mujeres del SERNAM, $50 \%$ son dueñas de casa y $37,5 \%$ eran trabajadoras; en cuanto a las mujeres del CESFAM, $64,29 \%$ eran dueñas de casa y $28,57 \%$ eran trabajadoras (Tabla 2). 
Tabla 1. Comparación de la versión original del instrumento WAST con la versión adaptada según los resultados de la prueba de jueces y grupo focal

\begin{tabular}{|c|c|}
\hline Wast: Versión original & Wast: Versión adaptada \\
\hline $\begin{array}{l}\text { 1. En general, ¿cómo describiría usted su relación con su } \\
\text { pareja? } \\
\text { o Mucha tensión } \\
\text { o Alguna tensión } \\
\text { o Sin tensión }\end{array}$ & $\begin{array}{l}\text { 1. En general, ¿cómo describiría usted su relación con su } \\
\text { pareja? } \\
\text { o Estresante } \\
\text { o Un poco estresante } \\
\text { o Nada estresante }\end{array}$ \\
\hline $\begin{array}{l}\text { 2. Usted y su pareja resuelven sus discusiones (argumentos) } \\
\text { con: } \\
\text { o Mucha dificultad } \\
\text { o Alguna dificultad } \\
\text { o Sin dificultad }\end{array}$ & $\begin{array}{l}\text { 2. Usted y su pareja resuelven sus discusiones con: } \\
\text { o Mucha dificultad } \\
\text { o Algo de dificultad } \\
\text { o Sin dificultad }\end{array}$ \\
\hline $\begin{array}{l}\text { 3. Al terminar las discusiones usted ise siente decaída o } \\
\text { mal con usted misma? } \\
\text { o Muchas veces } \\
\text { o A veces } \\
\text { o Nunca }\end{array}$ & $\begin{array}{l}\text { 3. Al terminar las discusiones usted ise siente decaída o } \\
\text { mal con usted misma? } \\
\text { o Muchas veces } \\
\text { o A veces } \\
\text { o Nunca }\end{array}$ \\
\hline $\begin{array}{l}\text { 4. ¿Las discusiones terminan en golpes, patadas, o empujo- } \\
\text { nes? } \\
\text { o Muchas veces } \\
\text { o A veces } \\
\text { o Nunca }\end{array}$ & $\begin{array}{l}\text { 4. ¿Las discusiones terminan en golpes, patadas, o empujo- } \\
\text { nes? } \\
\text { o Muchas veces } \\
\text { o A veces } \\
\text { o Nunca }\end{array}$ \\
\hline $\begin{array}{l}\text { 5. ¿Siente miedo de lo que su pareja diga o haga? } \\
\text { o Muchas veces } \\
\text { o A veces } \\
\text { o Nunca }\end{array}$ & $\begin{array}{l}\text { 5. ¿Hay situaciones en las cuales ha sentido miedo de las } \\
\text { reacciones de su pareja? } \\
\text { o Muchas veces } \\
\text { o A veces } \\
\text { o Nunca }\end{array}$ \\
\hline $\begin{array}{l}\text { 6. Su pareja ¿ha abusado de usted físicamente? } \\
\text { o Muchas veces } \\
\text { o A veces } \\
\text { o Nunca }\end{array}$ & $\begin{array}{l}\text { 6. Su pareja ¿controla el dinero que usted gasta, o la obliga } \\
\text { a realizar trabajo en exceso? } \\
\text { o Muchas veces } \\
\text { o A veces } \\
\text { o Nunca }\end{array}$ \\
\hline $\begin{array}{l}\text { 7. Su pareja ¿ha abusado de usted emocionalmente? } \\
\text { o Muchas veces } \\
\text { o A veces } \\
\text { o Nunca }\end{array}$ & $\begin{array}{l}\text { 7. Su pareja ¿la insulta, garabatea, grita, humilla y descalifi- } \\
\text { ca verbalmente? } \\
\text { o Muchas veces } \\
\text { o A veces } \\
\text { o Nunca }\end{array}$ \\
\hline $\begin{array}{l}\text { 8. ¿Su pareja ha abusado de usted sexualmente? } \\
\text { o Muchas veces } \\
\text { o A veces } \\
\text { o Nunca }\end{array}$ & $\begin{array}{l}\text { 8. ¿Se ha sentido obligada a tener relaciones sexuales con } \\
\text { su pareja para evitar problemas? } \\
\text { o Muchas veces } \\
\text { o A veces } \\
\text { o Nunca }\end{array}$ \\
\hline
\end{tabular}

Al realizar el análisis comparativo de las respuestas de ambos grupos, el grupo de mujeres víctimas de violencia mostraron mayor porcentaje de respuestas que connotaban la presencia de violencia en la relación de pareja en todos los aspectos indagados, mientras que en el grupo de usuarias del CESFAM, en su gran mayoría, refi- rieron no tener una relación estresante, resolvían los conflictos sin dificultes y no tenían miedo a las reacciones de su pareja (Tabla 3 ).

Para estimar el cálculo de punto de corte, sensibilidad, especificidad y alfa de Cronbach en el grupo sin violencia, de un total de 28 mujeres, el puntaje mínimo alcanzado fue de 8 
Tabla 2. Características sociodemográficas

\begin{tabular}{|c|c|c|}
\hline Variable & Mujeres sin violencia & Mujeres con certeza de violencia \\
\hline \multicolumn{3}{|l|}{ Estado civil } \\
\hline Casada & $15(53,57)$ & $10(62,50)$ \\
\hline Divorciada & $0 \quad(0)$ & $2(12,50)$ \\
\hline Soltera & $0 \quad(0)$ & $0 \quad(0)$ \\
\hline Conviviente & $9(32,14)$ & $2(12,50)$ \\
\hline Pololeando & $4(14,29)$ & $2(12,50)$ \\
\hline Total & $28(100 \%)$ & $16(100 \%)$ \\
\hline \multicolumn{3}{|l|}{ Edad } \\
\hline $18-24$ & $7(25,00)$ & $0 \quad(0)$ \\
\hline $25-34$ & $5(17,85)$ & $4(25,00)$ \\
\hline $35-44$ & $5(17,85)$ & $4(25,00)$ \\
\hline $45-54$ & $4(14,28)$ & $4(25,00)$ \\
\hline $55-64$ & $7 \quad(25,00)$ & $1 \quad(6,25)$ \\
\hline$>65$ años & & $3(18,75)$ \\
\hline Total & $28(100 \%)$ & $16(100 \%)$ \\
\hline \multicolumn{3}{|l|}{ Residencia } \\
\hline Recoleta & $28(100)$ & $0 \quad(0)$ \\
\hline Conchalí & $0 \quad(0)$ & $16(100)$ \\
\hline Total & $28(100 \%)$ & $16(100 \%)$ \\
\hline \multicolumn{3}{|l|}{ Nacionalidad } \\
\hline Chilena & $27 \quad(96,43)$ & $16(100)$ \\
\hline Peruana & $1 \quad(3,57)$ & $0 \quad(0)$ \\
\hline Total & $28(100 \%)$ & $16(100 \%)$ \\
\hline \multicolumn{3}{|l|}{ Ingresos } \\
\hline $0-71.788$ & $0 \quad(0)$ & $5 \quad(31,25)$ \\
\hline $71.789-120.229$ & $15 \quad(53,57)$ & $3(18,75)$ \\
\hline $120.230-184.909$ & $3(10,71)$ & $3(18,75)$ \\
\hline 184.910-337.775 & $8(28,57)$ & $4(25,00)$ \\
\hline 337.776-sin Límites & $2(7,14)$ & $1 \quad(6,25)$ \\
\hline Total & $28(100 \%)$ & $16(100 \%)$ \\
\hline \multicolumn{3}{|l|}{ Escolaridad } \\
\hline Básica incompleta & $3(10,71)$ & $4(25,00)$ \\
\hline Básica completa & $6(21,43)$ & $0 \quad(0)$ \\
\hline Media incompleta & $5(17,86)$ & $4(25,00)$ \\
\hline Media completa & $7 \quad(25,00)$ & $3(18,75)$ \\
\hline Enseñanza superior & $3(10,71)$ & $2(12,50)$ \\
\hline Profesional completa & $4(14,29)$ & $3(18,75)$ \\
\hline Total & $28(100 \%)$ & $16(100 \%)$ \\
\hline \multicolumn{3}{|l|}{$\mathrm{n}$ de hijos } \\
\hline 0 & $2 \quad(7,14)$ & $0 \quad(0)$ \\
\hline 1 & $9(32,14)$ & $2(12,50)$ \\
\hline 2 & $7 \quad(25,00)$ & $3(18,75)$ \\
\hline 3 & $4(14,29)$ & $10(62,5)$ \\
\hline 4 & $2 \quad(7,14)$ & $1 \quad(6,25)$ \\
\hline 5 & $4(14,29)$ & $0 \quad(0)$ \\
\hline Total & $28(100 \%)$ & $16(100 \%)$ \\
\hline \multicolumn{3}{|l|}{ Ocupación } \\
\hline Trabajador dependiente & $6(21,43)$ & $2(12,50)$ \\
\hline Dueña de casa & $18(64,29)$ & $8(50,00)$ \\
\hline Trabajador independiente & $2 \quad(7,14)$ & $4(25,00)$ \\
\hline Cesante & $1 \quad(3,57)$ & $2(12,50)$ \\
\hline Estudiante & $1 \quad(3,57)$ & $0 \quad(0)$ \\
\hline Total & $28(100 \%)$ & $16(100 \%)$ \\
\hline
\end{tabular}


Tabla 3. Resultados de la aplicación del instrumento WAST en cada muestra de mujeres, específico por cada pregunta (\%)

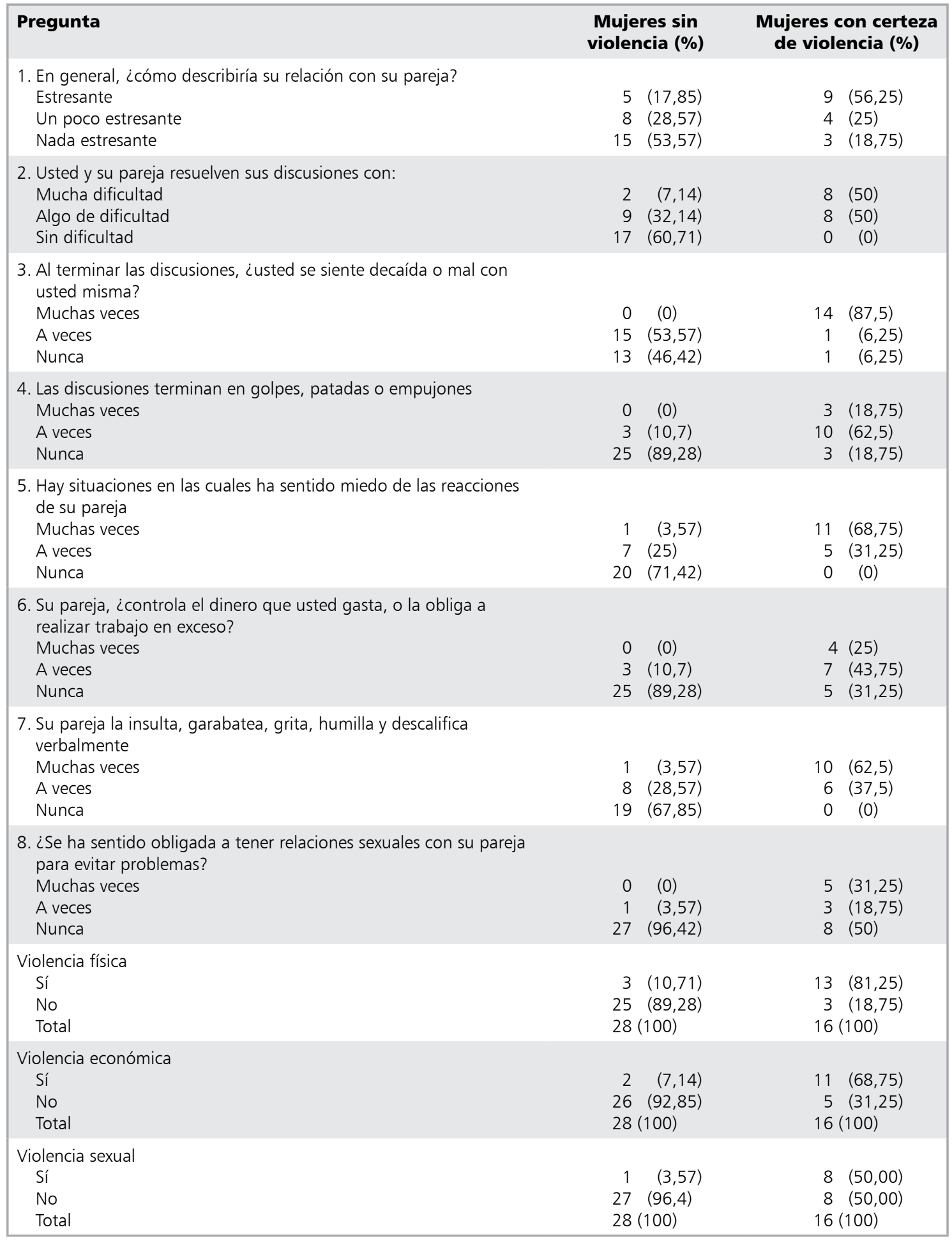


puntos (que corresponde al puntaje mínimo del instrumento), con una mediana de 10 puntos, y un máximo de 15 puntos. En la muestra de mujeres con violencia, el puntaje mínimo fue de 15 puntos, con una mediana de 19 puntos, y el puntaje máximo alcanzado fue de 24 puntos (que corresponde al puntaje máximo del instrumento) (Tabla 4). La confiabilidad del instrumento aplicado a las mujeres en estudio (alfa de Cronbach) fue de 91,5\%. Respecto a la capacidad de discriminación del instrumento, se encontró un área bajo la curva ROC de 99,6\% (IC: 98,6-100\%). La sensibilidad, especificidad y el LR+ para el punto de corte de 15 , se obtuvo $100 \%$ de sensibilidad, 96,4\% de especificidad y un LR+ de 28 (Tabla 4 y Figura 1).

Tabla 4. Puntajes finales de mujeres sin violencia y mujeres con certeza de violencia

\begin{tabular}{|lcccccc|}
\hline Muestra & Total & Mínimo & P25 & P50 & P75 & Máximo \\
\hline Mujeres sin violencia & 28 & 8 & 8 & 10 & 13 & 15 \\
\hline Mujeres con certeza de violencia & 16 & 15 & 16 & 19 & 21 & 24 \\
\hline Total & 44 & 8 & 9 & 13 & 18 & 24 \\
\hline
\end{tabular}
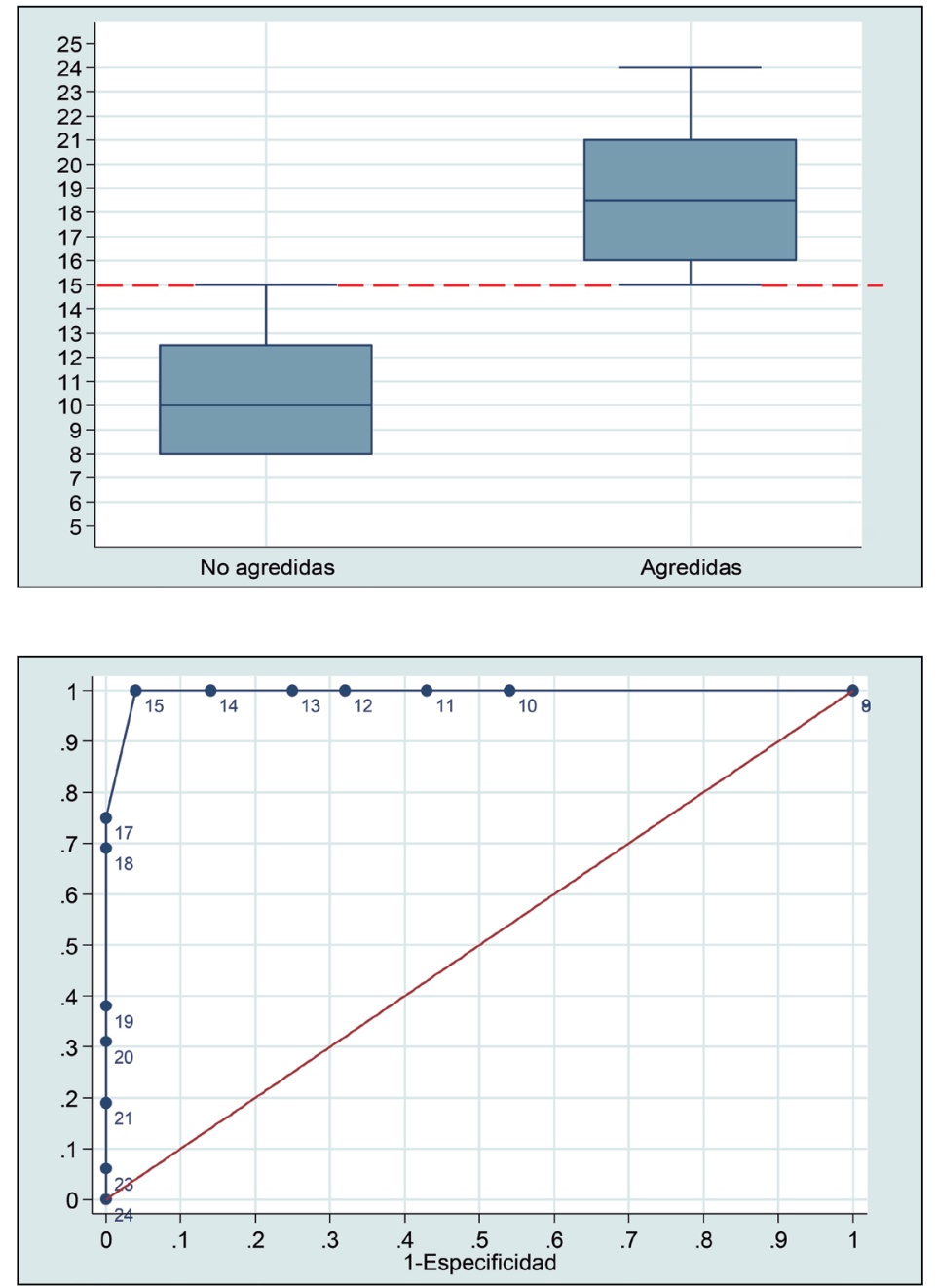

Figura 1. Distribución del puntaje WAST según condición de agredida.

Figura 2. Curva ROC de la discriminación del WAST a violencia doméstica. 


\section{Discusión}

Desde las políticas estatales se ha priorizado el tema de la violencia hacia la mujer por parte de su pareja, concentrando los esfuerzos en procurar una legislación adecuada, implementar programas de atención a víctimas y avanzar en sistemas de coordinación intersectorial. Sin embargo, un gran desafío sigue siendo el desarrollo de una política consistente y efectiva de prevención de la violencia de género ${ }^{20}$.

Parte de las recomendaciones internacionales para abordar la VD, que constituye un reconocido problema de salud pública ${ }^{20-22}$, es contar con una herramienta de tamizaje de detección precoz y aplicación rutinaria en APS, considerado el contexto idóneo para poner en marcha acciones que pronto incorporen las necesidades encotradas a programas y protocolos de respuesta y seguimiento. La literatura destaca este alcance como el primer paso para comenzar a abordar esta temática ${ }^{13}$, de ello se desprende la importancia de seleccionar un instrumento adecuado dentro de los que existen, autoaplicativo, que pueda ser utilizado a gran escala, de fácil comprensión y que haya sido aplicado con éxito en contexto y poblaciones similares, tal como entendemos que es el WAST ${ }^{13}$.

Utilizando este instrumento se pudo establecer, en relación a los resultados en la caracterización sociodemográfica de las mujeres con certeza de violencia y sin violencia, de acuerdo a lo encontrado en la literatura no se presentaron diferencias significativas ${ }^{1,22,23}$. En cuanto a la versión culturalmente adaptada del instrumento WAST, permite calificarlo como una herramienta diagnóstica altamente adecuada para la detección de VD, esto sustentado por sus valores de sensibilidad $100 \%$ y especificidad $96,43 \%$, valores que superan la validación de la versión hispana cuya sensibilidad fue de $89 \%$ y especificidad de $94 \%{ }^{13}$. Los valores del $\mathrm{LR}+$ obtenidos son también consistente con su alto valor diagnóstico de violencia ${ }^{23}$, adicionalmente, al incorporar la pregunta de violencia económica, la confiabilidad del instrumento aumentó ligeramente a 91,5\%, respecto del obtenido en la versión hispana de $91 \%$, lo cual lo califica como de excelente confiabilidad $^{13}$, aportándole un componente que ha sido evaluado en otros instrumentos internacionales de cribado de $\mathrm{VD}^{8,9}$.
En comparación a otras investigaciones que han validado el WAST, en este estudio se han conseguido resultados similares, logrando clasificar con puntajes más altos a las mujeres del grupo con violencia que a las mujeres sin violencia ${ }^{13,24,25}$.

La adaptación cultural local del instrumento WAST resultó tener una alta sensibilidad y especificidad al momento de detectar mujeres víctimas de VD utilizando el puntaje de corte de 15 puntos, por lo que esta herramienta puede ayudar en la discriminación de mujeres en las que se sospeche violencia, es importante destacar que un puntaje final menor a 15 puntos no implicará necesariamente que una mujer no sea víctima de alguna forma de violencia.

Es necesario abordar el problema de la VD desde diferentes frentes, la literatura reconoce el rol del sector salud, principalmente en la detección precoz, respondiendo a las recomendaciones internacionales, este instrumento puede ser una excelente herramienta de tamizaje que ponga en marcha un flujograma de acción articulado que permita dar seguimiento y apoyo a los casos detectados, por otra parte, realizar la adaptación cultural de un instrumento ya existente y utilizado a nivel mundial, permite, además, comparar indicadores de violencia en los distintos lugares donde se utilice $e^{9,21}$.

\section{Limitaciones del estudio}

La violencia en cualquiera de sus formas resulta un tema complejo y sensible, indagar sobre ella posiblemente constituya una limitación para las mujeres en querer exponerla, pudiendo esto traducirse en un subreporte. Adicionalmente, el formato Likert del instrumento también podría ofrecer dificultades de comprensión e interpretación, especialmente en población de baja escolaridad $^{26}$. Sin embargo, aun teniendo en consideración estas limitaciones, la validación cultural del instrumento mostró tener una buena capacidad de discriminación, rendimiento y confiabilidad.

Agradecimientos: Agradecemos sinceramente a todas las mujeres que generosamente colaboraron con su valiosa participación en este estudio. También agradecemos a SERNAM, SENAME, Ilustre Municipalidad de Recoleta y los CESFAM Cristo Vive y Dr. Patricio Hevia, instituciones que generosamente otorgaron todas las facilidades para la realización este trabajo. 


\section{Referencias}

1. WHO, Department of Reproductive Health and Research, London School of Hygiene and Tropical Medicine, South African Medical Research Council. Global and regional estimates of violence against women. Prevalence and health effects of intimate partner violence and non-partner sexual violence [Internet]. Ginebra 2013. Disponible en: http://www.who.int/reproductivehealth/ publications/violence/9789241564625/en/

2. Bott S, Guedes A, Goodwin M, Adams J. Violencia contra las mujeres en América Latina y el Caribe. Análisis comparativo de datos poblacionales de 12 países [Internet]. Washington DC: OPS, OMS; 2014. Disponible en: file://C:/Users/Usuario/Downloads/ViolenciaEspa\%C3\%B1ol_2.4-WEB\%20(2).pdf.

3. Rojas X, Peyrin C, Pezoa P, Quevedo M. La violencia tiene mil caras. Guía para profesionales y comunicadores/ as de medios de comunicación en violencia contra las mujeres [Internet]. Santiago: OMS, UNFPA, SERNAM, DOMOS; 2011. Disponible en: http://estudios.sernam. cl/documentos/?eMjM3NTYzNw==-La_Violencia_tiene_Mil_Caras:_Guia_para_Profesionales_y_Comunicadores/as_de_Medios_de_Comunicaci\%C3\%B3n_en_ Violencia_Contra_las_Mujeres.

4. Calvin M, Matamala M, Eguiguren P, Gálvez T, Berlagoscky F, Díaz X, et al. Informe Monográfico 2007-2012. Violencia de Género en Chile. Observatorio de Equidad de Género en Salud [Internet]. Santiago: OPS, Escuela de Salud Pública Universidad de Chile, CEM, EPES; 2013. Disponible en: http://www.paho.org/chi/index. php?option $=$ com_docman\&view $=$ download \&category_slug=sistema-de-salud\&alias $=123$-violencia-de-genero-en-chile-informe-monografico-2007-2012\&Ite$\operatorname{mid}=1145$.

5. SERNAM. Plan nacional de violencia intrafamiliar en Chile noviembre 2012-diciembre 2013 [Internet]. Santiago: Unidad de prevención de la violencia contra la mujer programa Chile Acoge; 2014. Disponible en: http://www.observatoriogeneroyequidad.cl/images/ DocuNata/Plan_Nacional_2012-2013.pdf.

6. ONU. Objetivos de desarrollo sostenible [Internet]. Nueva York; 2017. Disponible en: http://www.un.org/ sustainabledevelopment/es/gender-equality/

7. Minmujeryeg. Misión y funciones [Internet]. Santiago; 2017. Disponible en: http://www.minmujeryeg.cl/sernameg/mision/

8. Vargas V, Ortiz S, Rodríguez J. Estudio valorización de las prestaciones de salud por violencia basada en género en el espacio intrafamiliar [Internet]. Santiago: OPS, OMS; 2006. Disponible en: http://158.109.129.18/
centreantigona/docs/articulos/Estudio\%20valorizaci\%C3\%B3n\%20de\%20las\%20prestaciones $\% 20 \mathrm{de} \% 20$ salud\%20por\%20violencia\%20basada\%20en\%20g\%C3\%A9nero\%20en\%20el\%20espacio\%20intrafamiliar. pdf.

9. Santos R, Matías M, de Camargo CL, Pereira N, Freire N, Diniz A. Sexualidad, embarazo y violencia doméstica: experiencias de adolescentes brasileñas. Index Enferm [Internet] 2014; 23 (3): 139-43. Disponible en: http://scielo.isciii.es/scielo.php?script=sci_arttext\&pi$\mathrm{d}=$ S1132-12962014000200005\&lng=es. http://dx.doi. org/10.4321/S1132-12962014000200005.

10. Escuela Andaluza de Salud Pública, Unidad de Apoyo investigación. Catálogo de instrumentos para cribado y frecuencia del maltrato físico, psicológico y sexual [Internet]. Madrid; Observatorio de Salud de la Mujer Dirección General de la Agencia de Calidad del Sistema Nacional de Salud Secretaría General de Sanidad Ministerio de Sanidad y Consumo; 2004. Disponible en: http://www.msssi.gob.es/organizacion/sns/planCalidadSNS/pdf/equidad/genero_vg_01.pdf.

11. Minsal. Orientaciones técnicas para la atención integral de la mujer en edad de climaterio en el nivel primario de la red de salud (APS) [Internet]. Santiago; 2014. Disponible en: http://web.minsal.cl/sites/default/files/ files/OTCLIMATERIOinteriorValenteindd04022014. pdf.

12. Minsal. Programa salud de la mujer [Internet]. Santiago: División programa de salud; 1997. Disponible en: http:// web.minsal.cl/wp-content/uploads/2015/09/Programade-Salud-de-la-Mujer-1997.pdf.

13. Collen F, Fogarty, Belle J. Screening for abuse in Spanish-speaking women. J Am Board Fam Pract [Internet] 2002; 15: 101-11.

14. García-Esteve L, Torres A, Navarro P, Ascaso C, Imaz ML, Herreras Z, et al. Validación y comparación de cuatro instrumentos para la detección de la violencia de pareja en el ámbito sanitario. Med Clin [Internet]. 2011; 137 (9): 390-7.

15. Escobar-Pérez J, Cuervo-Martínez A. Validez de contenido y juicio de expertos: una aproximación a su utilización. Avances en Medición [Internet] 2008; 6: 27-36. Disponible en: http://www.humanas.unal.edu. co/psicometria/files/7113/8574/5708/Articulo3_Juicio_de_expertos_27-36.pdf.

16. Ulin P, Robinson E, Tolley E. La recolección de datos cualitativos: La ciencia y el arte. [Internet]. Washington DC: OPS, Oficina Sanitaria Panamericana, Oficina Regional de la OMS; 2006. Pag 3 p. Disponible en: http://iris.paho.org/xmlui/bitstream/handle/123456789/729/9275316147.pdf? sequence $=1$. 
17. Salech F, Mery V, Larrondo F, Rada G. Estudios que evalúan un test diagnóstico: interpretando sus resultados. Rev Med Chile [Internet] 2008; 136 (9): 1208.

18. Basile K, Hertz M, Back S. Intimate partner violence and sexual violence victimization assessment instruments for use in health care settings. Version 1.0 [Internet]. Atlanta: Centers for Disease Control and Prevention, National Center for Injury Prevention and Control; 2007 (20-21 p) Disponible en: https://www.cdc.gov/ violenceprevention/pdf/ipv/ipvandsvscreening.pdf

19. Burgueño MJ, García Bastos JL, González-Buitrago JM. Las curvas ROC en la evaluación de las pruebas diagnósticas. Med Clin [Internet]. 1995; 104(17): 661-70.

20. Larraín S. La situación de violencia contra las mujeres en Chile. Legislación y políticas públicas. Documento de Naciones Unidas [Internet]. 2008. Disponible en: http:// webarchive.unesco.org/20161127124609/http://portal. unesco.org/geography/es/files/11343/12436345861Resumen_ejecutivo_del_estudio_la_situacion_de_violencia_contra_las_mujeres_en_Chile._Legislacion_y_politicas_publicas.pdf/Resumen\%2Bejecutivo\%2Bde$1 \% 2 \mathrm{Bestudio} \% 2 \mathrm{Bla} \% 2 \mathrm{Bsituacion} \% 2 \mathrm{Bde} \% 2 \mathrm{Bviolen}-$ cia\%2Bcontra\%2Blas\%2Bmujeres\%2Ben\%2BChile.\%2BLegislacion\%2By\%2Bpoliticas\%2Bpublicas.pdf.

21. Cronholm PF, Fogarty CT, Ambuel B, Harrison SL.
Intimate partner violence. Am Fam Physician [Internet]. 2011; 15; 83 (10): 1165-72.

22. O'Campo P, Kirst M, Tsamis C, Chambers C, Ahmad F. Implementing successful intimate partner violence screening programs in health care settings: evidence generated from a realist-informed systematic review. Soc Sci Med. [Internet] 2011; 72 (6): 855-66.

23. López-Angulo L. Guía metodológica para la asistencia a mujeres víctimas de violencia: Una propuesta de indicadores. MediSur [Internet] 2009; 7 (5): 62-83. Disponible en: http://scielo.sld.cu/scielo.php?script=sci_arttext\&pi$\mathrm{d}=$ S1727-897X2009000500013\&lng=es.

24. Ochoa Sangrador C, González de Dios J, Buñuel Álvarez JC. Evaluación de artículos científicos sobre pruebas diagnósticas. Evid Pediatr [Internet] 2007; 3: 24. Disponible en: http://archivos.evidenciasenpediatria. es/_1wdMqnwxv5io8MsQyAC1eTD-yhC55oeFnVaKIfqQAMMobaBfTeXebPdv_GxBpt9kTYCbyLaDm08.

25. Iskandar L, Braun K Katz A. Testing the Woman Abuse Screening Tool to Identify Intimate Partner Violence in Indonesia. J Interpers Violence [Internet] 2015; 30 (7): 1208-25.

26. Bernal H, Wooley S, Schensul JJ. The challenge of using Likert-type scales with low-literate ethnic populations. Nurs Res 1997;46:179-81. 